\title{
CONTRACTIVE MAPPINGS ON A PREMETRIC SPACE
}

\author{
C.Y. SHEN and A. SOUND \\ Mathematics and Statistics Department \\ Simon Fraser University \\ Burnaby, B.C., V5A IS6 CANADA \\ (Received April 30, 1984)
}

ABSTRACT. In this piper, we study the fixed point property of certain types of :ontractive mappings defined on a premetric space. The applications of these results to topological vector spaces and to metric spaces are also discussed.

KEY WORDS AND PHRASES. Premetric space, sequentially $\phi$-contractive mappings, geometric mean contraction, iteratively $\phi$-contractive mapping, topological vector space.

1980 MATHEMATICS SUBJECT CLASSIEICATION. $47 H 10$.

1. INTRODUCTION.

The classical Banach contraction mapping principle can be simply stated as follows :

Let $(X, d)$ be a complete metric space, and let $T: X \rightarrow X$. Suppose there exists a constant $\mathrm{k}, 0 \leqq \mathrm{k}<1$, such that

$$
d(T x, T y) \leqq k d(x, y)
$$

for all $\mathrm{x}, \mathrm{y} \in \mathrm{X}$. Then $\mathrm{T}$ has a unique fixed point. It is well known that this principle is central to many existence proofs in mathematical analysis. Since its publication, much work has been done to generalize this result by allowing $X$ to be a more general type of space than the metric space or by relaxing the estimate $d(T x, T y) \leqq k d(x, y)$. In one of his papers [1], Wong introduced the concept of local iterative contraction. His idea was based on the observation that the inequality $\mathrm{d}(\mathrm{Tx}, \mathrm{Ty}) \leqq \mathrm{k} d(\mathrm{x}, \mathrm{y})$ can be expressed as

$$
d(T x, T y) \leq \phi(d(x, y))
$$

if one defines $\phi:[0, \infty) \rightarrow[0, \infty)$ by $\phi(t)=k t$. Of course, one can then generalize Banach's result by replacing the function $\phi(t)=k t$ with some more general type of function.

On the other hind, Kasahara [2] proved, in 1968, the Banach contraction principle for premetric spaces. Roughly speaking, a premetric space is different from a metric space by not having the symmetry property and the property that 
$\mathrm{d}(\mathrm{x}, \mathrm{y})>0$ if $\mathrm{x} \neq \mathrm{y}$. Kasahara demonstrated in his paper that there exists a $r$ lose connection between the premetric spaces and a certain class of subsets of a topological vector space (see next section). As a consequence, he showed that his result implied a fixed point theorem by Dubinsky for topological vector space.

In this paper, we take $\phi$ to be a monotone increasing function such that the series $\sum \phi^{k}(t)$ of its iterations converges for all $t>0$. This characterization if $\phi$ allows us to prove that certain mappings defined on a premetric space have i he fixed point property. We introduce, in Section 2, the notion of locally iteratively p-contractive mappings, and prove that they have fixed points. The application of this result to mappings defined on a topological space is also . ontained in that section. In Section 3, we study the sequentially $\phi$-contractive rappings on a premetric space. This concept has its origin from a paper by Yun [3]. in his manuscript, Yun introduced the notion of geometric mean contraction for a :sequence of mappings defined on a metric space, and stated: any sequential mapping with geometric mean contraction of a complete non-empty metric space II snto $M$ has a unique fixed point in $M$. This theorem in its present form, nowever, is incorrect, and we have shown a modified version follows from our Theorem 3.1.

\section{LOCALLY ITERATIVISY CONTRACTIVE MAPPINGS.}

Let $X$ be a non-empty set, let $D$ be a subset of $X \times X$, and let $d$ be a non-negative real-valued function defined on $D$. The triple $(X, D, d)$ is called a premetric space if the following two conditions are satisfied:

i. for all $x \in X,(x, x) \in D$ and $d(x, x)=0$.

ii. if $(x, y),(y, z) \in D$, then $(x, z) \in D$ and $d(x, z) \equiv d(x, y)+d(y, z)$. For convenience, we denote the set of all positive integers by $I$. A sequence $\left\{x_{n}\right\}$ in $X$ is r-convergent (right-convergent) to $x \in X$ if $\left(x, x_{n}\right) \in D$ for all $\mathrm{n} \varepsilon \mathrm{N}$ and $\mathrm{d}\left(\mathrm{x}, \mathrm{x}_{\mathrm{n}}\right) \rightarrow 0$ as $\mathrm{n} \rightarrow \infty$. A premetric space is $r$-separated if the limit of every $r$-convergent sequence is unique. A sequence $\left\{x_{n}\right\}$ in $X$ is a $r$-Cauchy sequence if for all $\ell, m \in \mathbb{N}$ with $\ell \leqq m,\left(x_{\ell}, x_{m}\right) \in D$ and $d\left(x_{\ell}, x_{m}\right) \rightarrow 0$ as $\mathrm{m} \rightarrow \infty$. A premetric space is $r$-complete if every $r$-Cauchy sequence in $X$ is $r$-convergent to some point in $X$. Similarly, one can define $\ell$-convergent sequences, $\ell$-Cauchy sequences, etc.: for example, a sequence $\left\{x_{n}\right\}$ in $X$ is $\ell$-convergent to $x \in X$ if $\left(x_{n}, x\right) \in D$ for all $n \in N$ and $d\left(x_{n}, x\right) \rightarrow 0$ as $n \rightarrow \infty$. By letting $x=[0, \infty), D=\{(x, x): x \in x\} \cup\{(1, x): 0 \leqq x \leqq 1\}$, and $d(x, y)=|x-y|$, we see that the sequence $\left\{1-\frac{1}{n}\right\}$ is $r$-convergent to 1 but it is not $r$-Cauchy. Thus, in a premetric space, not every $r$-convergent sequence is a r-Cauchy sequence. Next let $X$ be any non-empty set and let $D=X \times X$. If we let $d=0$, then every sequence in $X$ is $r$-convergent but the limit is not unique.

In an early paper by Kasahara [2], the author provided an interesting example of a premetric space which illustrated the close connection between the premetric spaces and the topological vector spaces. We shall describe this example here. Let $B$ be a non-empty, bounded, star-shaped (i.e., $\lambda B \subset B$ for all $0 \leqq \lambda \leqq 1$ ), convex subset of a linusdorff topological vector space L. Let $D$ be a subset of $E \times E$ such that $(x, y) \varepsilon D$ if and only if $x-y \in \lambda B$ for some real $\lambda>0$. For each pair $(x, y) \sim D$, we define $d(x, y)=\inf \{\lambda>0: x-y \varepsilon \lambda B\}$. Then 
$(E, D, d)$ is an $r$-separated and $\ell$-separated premetric space. It is easy to see that $(E, D, d)$ is a premetric space. To show that $E$ is $r$-separated, we follow Kasahara's argument by first noting that for every neighborhood $U$ of 0 in $E$, there exists a circled neighborhood $V$ (i.e., $\lambda V \subset V$ for all $|\lambda| \leqq 1$ ) such that $V+V \subset U$. Suppose $\left\{x_{n}\right\}$ is a sequence in $E$ which $r$-converges to $x$ and $\mathrm{y}$. Take $\mathrm{a} \delta>0$ with $\delta \mathrm{B}<\mathrm{V}$. Then $\mathrm{x}-\mathrm{x}_{\mathrm{n}}$ and $\mathrm{y}-\mathrm{x}_{\mathrm{n}}$ belong to $\delta \mathrm{B}$ for sufficiently large $n$. Since $V$ is circle, we have $x-y=\left(x_{n}-x\right)+\left(-y+x_{n}\right)$ $\varepsilon \mathrm{V}+\mathrm{V} \subset \mathrm{U}$. But $\mathrm{E}$ is Hausdorff, it follows that $\mathrm{x}=\mathrm{y}$.

Let $\phi$ be a function which maps the non-negative reals into itself such that $\phi$ is monotone increasing and $\sigma(t)=\sum^{\infty} \phi^{k}(t)$ converges for all $t>0,\left(\phi^{k}(t)\right.$ converges for all $t>0,\left(\phi^{0}(t)=t, \phi^{k}(t)=\phi\left(\phi^{k-1}(t)\right)\right.$.

DEFINITION 2.1. Let $(X, D, d)$ be a premetric space. A mapping $T: X \rightarrow X$ is said to be iteratively $\phi$-contractive at $z \varepsilon X$ if

i. $\left(\mathrm{T}^{\mathrm{m}}, \mathrm{z}, \mathrm{T}^{\mathrm{n}} \mathrm{z}\right) \in \mathrm{D}$ for all $\mathrm{m}, \mathrm{n} \in \mathrm{N}_{0}=\{0,1,2, \ldots\}$, and

ii. there exists a positive integer $n(z)$ such that for all $(x, y) \varepsilon D$ and $\left(\mathrm{T}^{\mathrm{n}(\mathrm{z})} \mathrm{x}, \mathrm{T}^{\mathrm{n}(\mathrm{z})} \mathrm{y}\right) \in \mathrm{D}$,

$$
a\left(T^{n(z)} x, T^{n(z)} y\right) \equiv \phi(d(x, y)) \text {. }
$$

Note that from part (i) of the above definition, we have $\left(T^{n(z)} x, m^{n(z)} y\right) \varepsilon D$ for all $x, y \in\left\{T^{n} z: n \in N_{0}\right\}$. Next, we prove

LEMMA 2.1. Let $\phi$ be given as above. Then $\phi(t)<t$ for all $t>0$ and $\phi$ is continuous at 0 with $\phi(0)=0$.

PROOF. Suppose there exists a $t>0$ such that $\phi(t) \geqq t$. Since $\phi$ is monotone increasing, $\phi^{k}(t) \leqq t>0$ for all $k \in N$. Hence, $\underset{\infty}{\lim } \phi^{\mathrm{k}}(\mathrm{t}) \leqq \mathrm{t}>0$ which contradicts the fact that $\sum_{0} \phi^{\mathrm{k}}(\mathrm{t})<\infty$.

The second part follows easily from the inequality

$$
0 \leqq \phi(0) \leqq \lim _{t \rightarrow 0^{+}} \phi(t) \leqq \lim _{t \rightarrow 0^{+}} t .
$$

Before we state our main theorem for this section, we point out that if $Y$ is a subset of a premetric space $(X, D, d)$, then $\left(Y, D^{\prime}, d^{\prime}\right)$ is also a premetric space with $D^{\prime}=D \cap(Y \times Y)$ and $d^{\prime}=\left.d\right|_{D^{\prime}}$.

THEOREM 2.1. Let $(X, D, d)$ be a r-separated premetric space and let $T: X \rightarrow X$ be iteratively $\phi$-contractive at $z \varepsilon X$. If $X_{0}=\left\{x \in X:\left(T^{n} x, z\right) \varepsilon D\right.$ for all $\left.\mathrm{n} \varepsilon \mathrm{N}_{0}\right\}$ is $r$-complete, then $\mathrm{T}$ has a unique fixed point in $\mathrm{X}_{0}$ and the successive iterations $u_{k}=T u_{k-1}, u_{0}=z$, are $r$-convergent to the fixed point.

PROOF. Let $S=T^{n(z)}$ and define recursively $v_{0}=z, v_{k}=S v_{k-1}$. We shall show that $\left\{v_{k}\right\}$ forms a r-Cauchy sequence in $x_{0}$. From condition (i) on $T$ it is clear that $v_{k} \varepsilon X_{0}$ for all $k \in N_{0}=\{0,1,2, \ldots\}$ and $\left(v_{m}, v_{n}\right) \varepsilon D$ for all $m, n \in N_{0}$. By condition ( $\left.i i\right)$ on $T$, we have

$$
d\left(v_{m+k}, v_{m}\right)=\phi^{m}\left(d\left(v_{k}, v_{0}\right)\right), \quad\left(m, k \in N_{0}\right) \text {. }
$$

Since

$$
\begin{aligned}
d\left(v_{k}, v_{0}\right) & \leqq d\left(v_{k}, v_{k-1}\right)+\ldots+d\left(v_{1}, v_{0}\right) \\
& \cong \phi^{k-1}\left(d\left(v_{1}, v_{0}\right)\right)+\ldots+d\left(v_{1}, v_{0}\right) \\
& \leqq \sigma(d(S z, z))
\end{aligned}
$$


and $\phi$ is monotone increasing, we obtain

$$
\mathrm{d}\left(\mathrm{v}_{\mathrm{m}+\mathrm{k}}, \mathrm{v}_{\mathrm{m}}\right) \leqq \phi^{\mathrm{m}}(\sigma(\mathrm{d}(\mathrm{Sz}, \mathrm{z}))
$$

for all $\mathrm{m}, \mathrm{k} \in \mathrm{N}_{0}$. Using the fact that $\phi^{\mathrm{m}}(\mathrm{t}) \rightarrow 0$ for all $t \geqq 0$ as $\mathrm{m} \rightarrow \infty$, we have shown that $\left\{v_{m}\right\}$ is a r-Cauchy sequence in $x_{0}$.

Since $x_{0}$ is $r$-complete, the sequence $\left\{v_{k}\right\}$ is $r$-convergent to some point $\mathrm{x}_{0} \varepsilon \mathrm{x}_{0}$. It follows from the inequality $\mathrm{d}\left(\mathrm{Sx_{0 }}, \mathrm{v}_{\mathrm{k}+1}\right) \leqq \phi\left(\mathrm{d}\left(\mathrm{x}_{0}, \mathrm{v}_{\mathrm{k}}\right)\right)$ for all $k \varepsilon N_{0}$ and the fact that $\phi$ is continuous at 0 with $\phi(0)=0$, the sequence $\left\{v_{k}\right\}$ is r-convergent to $S x_{0}$. Since $X$ is r-separated, $S x_{0}=x_{0}$.

Because $\left(\mathrm{T}^{\mathrm{i}} \mathrm{x}_{0}, \mathrm{z}\right) \in \mathrm{D}$ and $\left(\mathrm{z}, \mathrm{v}_{\mathrm{k}}\right) \in \mathrm{D}$ for all $\mathrm{i}, \mathrm{k} \varepsilon \mathrm{N}_{0}$, we have $\left(\mathrm{T}^{\mathrm{i}} \mathrm{x}_{0}, \mathrm{v}_{\mathrm{k}}\right) \in \mathrm{D}$ for all $\mathrm{i}, \mathrm{k} \in \mathrm{N}_{0}$. In particular,

$$
d\left(T x_{0}, v_{k}\right)=d\left(T S^{k} x_{0}, S^{k} z\right) \leqq \phi^{k}\left(d\left(T x_{0}, z\right)\right)
$$

for all $\mathrm{k} \in \mathrm{N}_{0}$. Thus, $\left\{\mathrm{v}_{\mathrm{k}}\right\}$ is $\mathrm{r}$-convergent to $\mathrm{Tx}_{0}$; whence, $\mathrm{Tx}_{0}=\mathrm{x}_{0}$.

Now suppose there exists another $y_{0}$ in $x_{0}$ such that $T y_{0}=y_{0}$. Then

$$
\mathrm{d}\left(\mathrm{y}_{0}, \mathrm{v}_{\mathrm{k}}\right)=\mathrm{d}\left(\mathrm{S}^{\mathrm{k}} \mathrm{y}_{0}, \mathrm{~s}^{\mathrm{k}} \mathrm{z}\right) \leqq \phi^{\mathrm{k}}\left(\mathrm{d}\left(\mathrm{y}_{0}, \mathrm{z}\right)\right)
$$

which implies that $\mathrm{y}_{0}=\mathrm{x}_{0}$.

Finally, we will show that the sequence $\left\{u_{k}\right\}$ where $u_{0}=z, u_{k}=T u_{k-1}$ is r-convergent to the fixed point $x_{0}$. To this end, note that for all $\ell \geq n(z)$, $\ell=m n(z)+k$ for some $m, k \varepsilon N_{0}$. Thus, we have the following inequality:

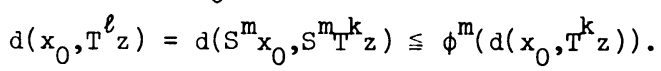

Let $\gamma=\max \cdot\left\{d\left(x_{0}, T^{k} z\right): k=0,1, \ldots, n(z)\right\}$. Then $d\left(x_{0}, T^{\ell} z\right) \leqq \phi^{m}(\gamma)$ which shows that $\left\{\mathrm{T}^{\ell} \mathrm{z}\right\}$ is $\mathrm{r}$-convergent to $\mathrm{x}_{0}$.

As an immediate consequence of Theorem 2.1, we have the following fixed point theorem in the setting of a topological vector space:

COROLLARY 2.1. Let $E$ be a Hausdorff topological vector space, and let $B$ be a bounded star-shaped convex subset of $E$. Let $T: E \rightarrow E$ be a mapping such that the following two conditions are satisfied:

i. there exists a $z \in \mathrm{E}$ such that for all $m, n \in N, T^{m} z-T^{n} z \varepsilon \lambda B$ for some $\lambda \geqq 0$.

ii. there exists a positive integer $n(z)$ such that for all $x, y, x-y \in \lambda B$ implies $T^{n(z)} x-T^{n(z)}$ y $\varepsilon \phi(\lambda) B$ where $\phi$ is given as before.

If $X_{0}=\left\{x \in E: T^{n} x-2 \in \lambda_{n} B\right.$ for some $\lambda_{n} \geqq 0$ and for all $\left.n \varepsilon N\right\}$ is sequentially complete, then $T$ has a unique fixed point in $X_{0}$.

3. SEQUENTIAL CONTRACTIVE MAPPIIGG.

Let $(X, D, d)$ be a premetric space and let $\left\{T_{i}: i \varepsilon N\right\}$ be a sequence of mappings from $X$ into $X$. If $T_{i} T_{j}=T_{j} T_{i}$ for all $i, j \in N$, then we say this sequence of mappings is commutative. For each positive integer $\mathrm{n}$, we define $T^{n+1}=T_{n+1} T^{n}$ with $T^{l}=T_{1}$. If $i$ is a positive integer less than or equal to $n$, by $T_{(i)}^{n}$, we mean

$$
T_{(i)}^{n}=T_{n} \cdots T_{i+1} T_{i-1} \cdots T_{1} \text {. }
$$

We shall adopt the convention that $T^{n}{ }_{(i)}=T^{n}$ if if $i>n$.

DEFINITION 3.1. A sequence $\left\{\mathrm{T}_{i}\right\}$ is said to be sequentially $\phi$-contractive if $(x, y) \in D$ implies that 
i. for all $i \in N,\left(T_{i} x, T_{i} y\right) \varepsilon D$ and $d\left(T_{i} x, T_{i} y\right) \leqq C_{i} d(x, y)$, and

ii. for all $n, i \varepsilon N$,

$$
d\left(T^{n}(i)^{x}, T^{n}(i)^{y)} \leqq \begin{cases}\phi^{n}(d(x, y)) & \text { if } i>n \\ C_{i} \phi^{n}(d(x, y)) & \text { if } i \leqq n\end{cases}\right.
$$

where $C_{i}^{\prime}$ s are constant and $\phi$ is a function as given in section 2 .

THEOREM 3.1. Let $X$ be a r-separated premetric space, and let $\left\{\mathrm{T}_{i}\right\}$ be a sequence of commutative, sequentially $\phi$-contractive mappings. If the set $Y=\left\{y:\left(T_{i} y, y\right) \varepsilon D\right.$ for all $\left.i \varepsilon N\right\}$ is r-complete and if there exists a z $\varepsilon Y$ such that $\sum_{l}^{\infty} \phi^{k-1}\left(t_{k}\right)<\infty$ where $t_{k}=d\left(T_{k} z, z\right)$ and $(y, z) \varepsilon D$ for all $y \in Y$, then there exists a unique $y_{0} \varepsilon Y$ such that $T_{i} y_{0}=y_{0}$ for all $i \varepsilon N$.

PROOF. First, we'll show that $\left\{\mathrm{T}^{\mathrm{n}} \mathrm{z}\right\}$ is a r-Cauchy sequence in $Y$. From the facts $\left(T_{n} z, z\right)$, $D$ for all $n \varepsilon N$ and $\left\{T_{i}\right\}$ is commutative, it follows that $\left\{T^{n} z\right.$ belong to $Y$ and $\left(T^{n+l} z, T^{n} z\right) \varepsilon D$ for all $n \varepsilon N$. Using the transitive property of $D$, we have, for all $m, n \varepsilon \mathbb{N}$ with $m \equiv n$,

$$
\begin{aligned}
d\left(T^{m} z, T^{n} z\right) & \leqq d\left(T^{m} z, T^{m-1} z\right)+\ldots+d\left(T^{n+1} z, T^{n} z\right) \\
& \equiv \phi^{m-1}\left(t_{m}\right)+\ldots+\phi^{n}\left(t_{n+1}\right) \\
& =\sum_{n}^{m-1} \phi^{k}\left(t_{k+1}\right)
\end{aligned}
$$

which goes to zero as $n \rightarrow \infty$. Thus, the sequence $\left\{\mathrm{T}^{\mathrm{n}} \mathrm{z}\right\}$ is $\mathrm{r}$-convergent to some point $\mathrm{y}_{0} \in \mathrm{Y}$.

Next, we claim that for each fixed $i \varepsilon N$, the sequence $\left\{T^{n}(i)^{z}\right\}$ is also a $r$-Cauchy sequence in $Y$. Moreover, it $r$-converges to $y_{0}$ (It turns out that the $r$-Cauchy property is not needed in our proof). It is easy to see that $\left\{T^{n}(i)^{z}\right\}$ is in $Y$. Now let $m, n \varepsilon N$ with $m \geq n \geq i$. We have

$$
\mathrm{d}\left(\mathrm{T}^{\mathrm{m}}(\mathrm{i})^{z, T^{\mathrm{n}}}(\mathrm{i})^{z}\right) \equiv \mathrm{C}_{i} \phi^{\mathrm{m}-1}\left(\mathrm{t}_{\mathrm{m}}\right)+\ldots+\mathrm{C}_{i} \phi^{\mathrm{n}\left(\mathrm{t}_{\mathrm{n}+1}\right)}
$$

which shows that $\left\{T^{n}(i)^{z}\right\}$ is a r-Cauchy sequence. Since $\left(T_{i} z, z\right) \varepsilon D$, we see that $\left(T^{n+1} z, T_{(i)}^{n+1} z\right) \in D$. Hence,

$$
\begin{aligned}
d\left(y_{0}, T_{(i)}^{n+1} z\right) & \leqq d\left(y_{0}, T^{n+1} z\right)+d\left(T^{n+1} z, T^{n+1} z\right) \\
& \leqq d\left(y_{0}, T^{n+1} z\right)+c_{i} \phi^{n+1}\left(d\left(T_{i} z, z\right)\right)
\end{aligned}
$$

which goes to zero as $\mathrm{n} \rightarrow \infty$.

Finally, $\left(\mathrm{y}_{0}, \mathrm{~T}^{\mathrm{r}} \mathrm{z}\right),\left(\mathrm{T}_{i} \mathrm{y}_{0}, \mathrm{y}_{0}\right) \in \mathrm{D}$ implies that $\left(\mathrm{T}_{i} \mathrm{y}_{0}, \mathrm{~T}^{\mathrm{n}} \mathrm{z}\right) \varepsilon \mathrm{D}$ and

$$
\begin{aligned}
& d\left(T_{i} y_{0}, T^{n} z\right)=d\left(T_{i} y_{0}, T^{n}(i) T_{i} z\right) \\
& \leq C_{i} d\left(y_{0}, T^{n}(i)^{z}\right)
\end{aligned}
$$

which goes to zero as $\mathrm{n} \rightarrow \infty$. Since $\mathrm{X}$ is $\mathrm{r}$-separated, we have $\mathrm{T}_{i} \mathrm{y}_{0}=\mathrm{y}_{0}$ for all i $\varepsilon$ IN.

To show $\mathrm{y}_{0}$ is unique, we proceed as follows: suppose there exists another $\mathrm{y}_{1} \in \mathrm{Y}$ such that $\mathrm{T}_{i} \mathrm{y}_{1}=\mathrm{y}_{1}$ for all $\mathrm{i}$. Then $\left(\mathrm{y}_{1}, \mathrm{z}\right) \varepsilon D$ which implies that

$$
\left(\mathrm{y}_{1}, \mathrm{~T}^{\mathrm{n}} \mathrm{z}\right)=\left(\mathrm{T}^{\mathrm{n}} \mathrm{y}_{1}, \mathrm{~T}^{\mathrm{n}} \mathrm{z}\right) \in \mathrm{D}
$$




$$
\mathrm{d}\left(\mathrm{y}_{1}, \mathrm{~T}^{\mathrm{n}} \mathrm{z}\right) \leqq \phi^{\mathrm{n}}\left(\mathrm{d}\left(\mathrm{y}_{1}, \mathrm{z}\right)\right) .
$$

Hence, $\left\{T^{n} z\right\}$ is $r$-convergent to $y_{1}$, and by $r$-separateness of $x$, we see that $\mathrm{y}_{1}=\mathrm{y}_{0}$.

One can obtain an immediate corollary of the above theorem if we first define DEFINITION 3.2. A sequence $\left\{T_{i}\right\}$ of self-mappings on a premetric space $X$ is said to be $\phi$-contractive if $(x, y) \varepsilon D$ implies that $\left(T_{i} x, T_{i} y\right) \varepsilon D$ and $\mathrm{d}\left(\mathrm{T}_{\mathrm{i}} \mathrm{x}, \mathrm{T}_{\mathrm{i}} \mathrm{y}\right) \leqq \phi(\mathrm{d}(\mathrm{x}, \mathrm{y}))$ for all $\mathrm{i} \varepsilon \mathrm{N}$ with $\phi$ as given before.

COROLLARY 3.1. Let $\left\{\mathrm{T}_{i}\right\}$ be a sequence of commutative $\phi$-contractive selfmappings defined on a $r$-separated premetric space $X$. If the set $Y=\left\{\mathrm{y}:\left(\mathrm{T}_{i} \mathrm{y}, \mathrm{y}\right) \varepsilon \mathrm{D}\right.$ for all $\left.i \varepsilon \mathrm{N}\right\}$ is $\mathrm{r}$-complete and if there exists a $\mathrm{z} \in \mathrm{Y}$ such that $\sum_{l}^{\infty} \phi^{k-1}\left(t_{k}\right)<\infty$ where $t_{k}=d\left(T_{k}, z, z\right)$ and $(y, z) \varepsilon D$ for all $y \varepsilon Y$, then there exists a unique $\mathrm{y}_{0} \varepsilon \mathrm{Y}$ such that $\mathrm{T}_{\mathrm{i}} \mathrm{y}_{0}=\mathrm{y}_{0}$ for all $\mathrm{i} \varepsilon \mathrm{IN}$.

PROOF. One only needs to observe that $\left\{\mathrm{T}_{i}\right\}$ is also sequentially $\phi$-contractive with $C_{i}=1$ for all $i$.

In a manuscript by Yun [3], the author introduced the notion of geometrical mean contraction for a sequence $\left\{\mathrm{T}_{i}\right\}$ of self-mappings defined on a metric space $X$. After a careful study of his work, we believe his definition and also the statement of Theorem 1 in his paper need to be modified. We shall give here our version.

DEFINITION 3.3. Let $(\mathrm{X}, \mathrm{d})$ be a metric space and let $\left\{\mathrm{T}_{i}\right\}$ be a sequence of self-mappings on $X$. We say that $\left\{T_{i}\right\}$ is a sequential mapping with geometric mean contraction if for each i $\varepsilon N$, we have

$$
\mathrm{d}\left(\mathrm{T}_{i} \mathrm{x}, \mathrm{T}_{\mathrm{i}} \mathrm{y}\right) \leqq \mathrm{r}_{\mathrm{i}} \mathrm{d}(\mathrm{x}, \mathrm{y}) \quad(\mathrm{x}, \mathrm{y} \in \mathrm{X})
$$

where $r_{i}$ is a positive constant, and, for all $n \varepsilon N$,

$$
\left(r_{1} \ldots r_{n}\right)^{1 / n}<G
$$

where $0 \leqq G<1$ is a constant.

Clearly, if $X$ is a metric space, then the set $Y$ in Theorem 3.1 is equal to $\mathrm{X}$. Also, if $\left\{\mathrm{T}_{i}\right\}$ is a sequential mapping with geometric mean contraction, then $\left\{\mathrm{T}_{i}\right\}$ is sequentially $\phi$-contractive provided we let $C_{i}=\max .\left\{r_{i}, \frac{1}{r_{i}}\right\}$ and $\phi(t)=G t, t \geqq 0$.

COROLUARY 3.2. Let $X$ be a complete metric space and let $\left\{T_{i}\right\}$ be a commutative sequential mapping with geometric mean contraction. If there exists an $x \in X$ such that the set $\left\{d\left(T_{i} x, x\right): i \varepsilon N\right\}$ is bounded, then $\left\{T_{i}\right\}$ has a unique common fixed point.

PROOF. Let $b$ be an upper bound of $\left\{d\left(T_{i} x, x\right): i \varepsilon N\right)$. Since $\phi$ is monotone increasing, we have

$$
\phi^{k-1}\left(t_{k}\right) \leqq \phi^{k-1}(b)
$$

where $t_{k}=d\left(T_{k} x, x\right)$. Hence, $\sum \phi^{k-1}\left(t_{k}\right)<\infty$, and the corollary now follows easily from Theorem 3.1.

We shall remark here the Corollary 3.2 remains valid if we replace the requirement that $\left\{\mathrm{T}_{i}\right\}$ be commutative by the following less stringent condition: $\left\{T_{i}\right\}$ is said to be weakly commutative if $T_{n+1} T^{n}=T^{n} T_{n+l}$ for all $n \varepsilon N$. 
ACKNOWLEDGEMLITT. This research is supported by the Natural Sciences and Engineering Research Council Grant A8511.

\section{REFEREIVCES}

1. WONG, J. Two extensions of the Banach contraction mapping principle, J. of Math. Analysis and Appl., Vol. 22, No. 2, 1968, p. 438-443.

2. KASAHARA, S. A remark on the contraction principle, Proc. Japan Acad., 44, 1968, p. 21-26.

3. YUN, Tian-quan. Fixed point theorems of sequential mapping with geometric mean contraction and its applications, Preprint. 


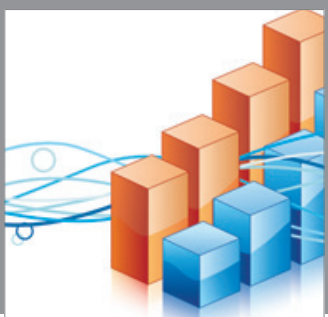

Advances in

Operations Research

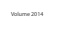

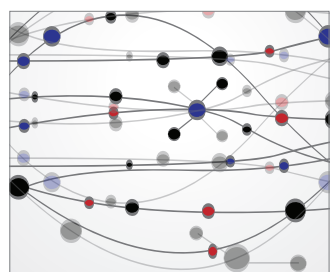

\section{The Scientific} World Journal
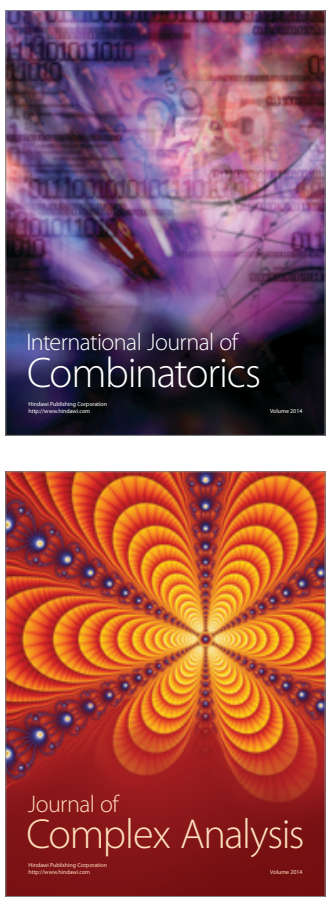

International Journal of

Mathematics and

Mathematical

Sciences
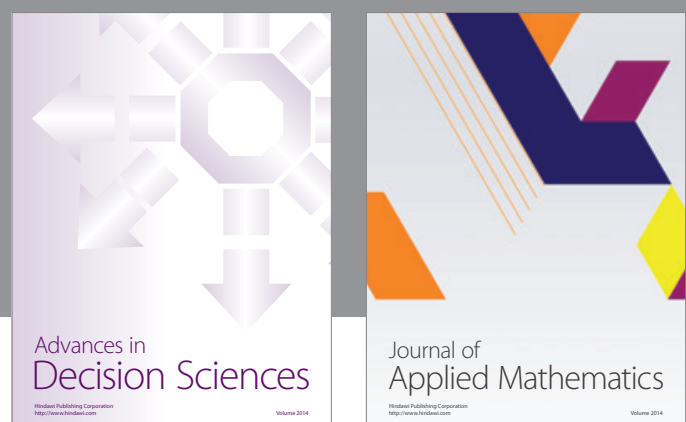

Journal of

Applied Mathematics
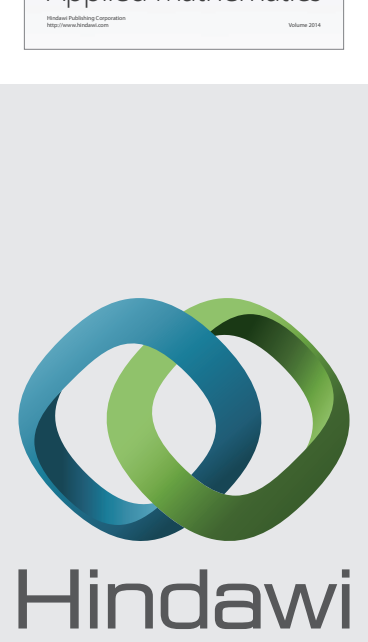

Submit your manuscripts at http://www.hindawi.com
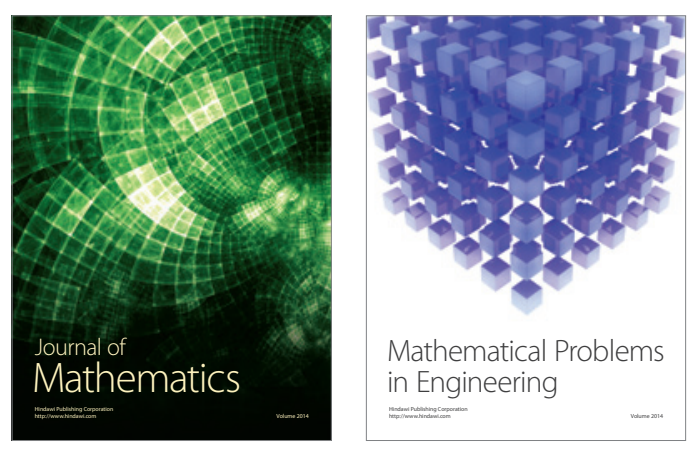

Mathematical Problems in Engineering
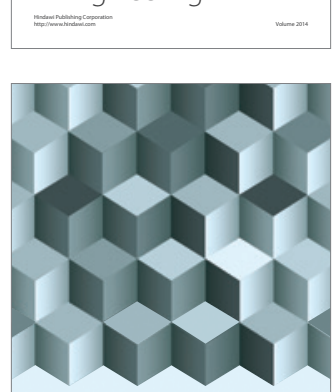

Journal of

Function Spaces
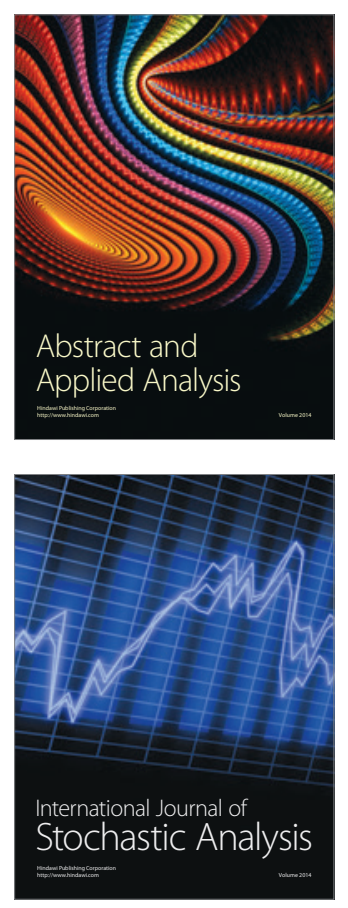

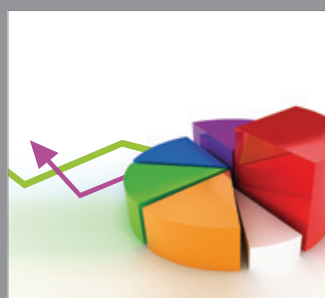

ournal of

Probability and Statistics

Promensencen
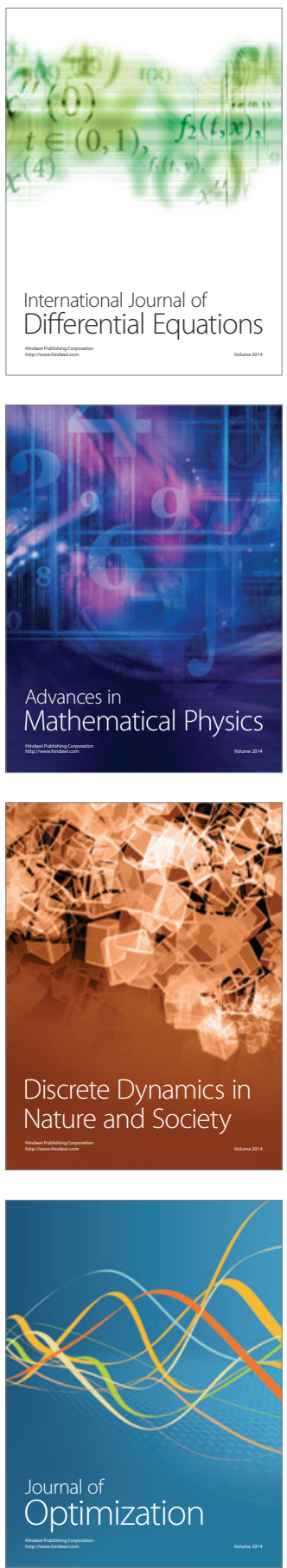